Abstract 85 Table 1 Characteristics of 501 patients with SLE and polyautoimmunity

\begin{tabular}{|c|c|}
\hline Variable & polyautoimmunity, $(n=501)$ \\
\hline \multicolumn{2}{|l|}{ Epidemiological characteristics } \\
\hline Sex (female), n (\%) & $473(94.4)$ \\
\hline Ethnicity, caucasian (\%) & $456(93.6)$ \\
\hline \multicolumn{2}{|l|}{ Age at disease diagnosis, mean (SD) } \\
\hline Age $>50$ years, $n(\%)$ & $134(15.4)$ \\
\hline \multicolumn{2}{|l|}{ Smoke } \\
\hline Non-smoker, n (\%) & $274(59.4)$ \\
\hline Ex-smoker, n (\%) & $120(26.0)$ \\
\hline Smoker, n (\%) & $67(14.5)$ \\
\hline \multicolumn{2}{|l|}{ Oral contraceptive } \\
\hline Non-oral contraceptive, n (\%) & $258(69.0)$ \\
\hline Exoral contraceptive, $n(\%)$ & $107(28.6)$ \\
\hline Oral contraceptive, n (\%) & $9(2.4)$ \\
\hline Family history , n (\%) & $60(15.7)$ \\
\hline \multicolumn{2}{|l|}{ Clinical characteristic } \\
\hline Disease duration (months), median(p75-p25) & $212.0(120.8-289.0)$ \\
\hline Malar rash, $\mathrm{n}(\%)$ & $253(50.8)$ \\
\hline Discoid rash, n (\%) & $94(19.1)$ \\
\hline Photosensitivity, n (\%) & $293(59.7)$ \\
\hline Oral ulcers, n (\%) & $218(44.4)$ \\
\hline Arthritis, $\mathrm{n}(\%)$ & $393(79.4)$ \\
\hline Pleuritis, n (\%) & $105(21.3)$ \\
\hline Pericarditis, n (\%) & $86(17.3)$ \\
\hline Renal disorder, $\mathrm{n}(\%)$ & $132(26.6)$ \\
\hline Neurological disorder, $\mathrm{n}(\%)$ & $45(9.1)$ \\
\hline hemolytic anemia, n (\%) & $49(9.9)$ \\
\hline leukopenia, n (\%) & $293(59.3)$ \\
\hline thrombocytopenia, n (\%) & $106(21.8)$ \\
\hline Sjögren's syndrome, n (\%) & $127(25.7)$ \\
\hline Antiphospholipid syndrome, n (\%) & $74(14.9)$ \\
\hline mixed connective tissue disease, $\mathrm{n}(\%)$ & $14(2.7)$ \\
\hline Raynaud's phenomenon, $\mathrm{n}(\%)$ & $226(45.8)$ \\
\hline Lupus Nephritis, n (\%) & $131(26.5)$ \\
\hline Pulmonary fibrosis, n (\%) & $25(5.0)$ \\
\hline Pulmonary hypertension, $\mathrm{n}(\%)$ & $17(3.4)$ \\
\hline \multicolumn{2}{|l|}{ Laboratory characteristic } \\
\hline ANA,$+ n(\%)$ & $497(99.0)$ \\
\hline Anti-DNA+, n (\%) & $350(71.0)$ \\
\hline Anti-sm +, n (\%) & $110(22.8)$ \\
\hline Anti-RNP, n (\%) & $164(34.1)$ \\
\hline Anti-ro, n (\%) & $193(39.9)$ \\
\hline Anti-la, n (\%) & $104(21.4)$ \\
\hline$A L+, n(\%)$ & $70(20.3)$ \\
\hline \multicolumn{2}{|l|}{ Therapeutic regimen } \\
\hline Glucocorticoids, n (\%) & $439(91.1)$ \\
\hline Methotrexate, n (\%) & $120(24.7)$ \\
\hline Hydroxychloroquine, n (\%) & $369(76.7)$ \\
\hline Azathioprine, $\mathrm{n}(\%)$ & $173(36.0)$ \\
\hline Cyclophosphamide, n (\%) & $95(19.7)$ \\
\hline Mycophenolate mofetilo, n (\%) & $60(12.4)$ \\
\hline Rituximab, n (\%) & $44(9.1)$ \\
\hline Inmunoglobulin, n (\%) & $23(4.8)$ \\
\hline
\end{tabular}

Background OBJECTIVE: Estimate the frequency of the association of SLE with other autoimmune diseases in a large Spanish cohort of patients with systemic lupus erythematosus (SLE) and investigate the main risk factors for polyatoimmunity.

Methods Design: RELESSER is a nationwide multicentre, hospitalbased registry of SLE patients. This is a cross-sectional study.
Patients: Unselected consecutive adult patients with SLE, classified according to the American College of Rheumatology (ACR) 1997 criteria. All patients had been attended upon and followed at Spanish rheumatology departments. The first patient was enrolled in October 2011 and the last in August 2012. Main outcome: Polyautoimmunity was defined as patients who fulfilled criteria for SLE and other autoimmune disease: (1) autoimmune thyroiditis (alteration of thyroid function with the presence of anti-thyroid autoantibodies), (2) other connective tissue disease (rheumatoid arthritis, systemic sclerosis or inflammatory myopathy) and (3) mixed connective tissue disease. Multiple autoimmune syndrome (MAS) was defined as patients who meet SLE criteria and at least two other autoimmune diseases. Other variables: Demographic and clinical variables, Sjogren's syndrome, antiphospholipid syndrome and family history of autoimmune systemic disease were collected. Statistical analysis: Descriptive, Chi-square test and ANOVA or KruskalWallis for comparison between groups of patients. Multiple logistic regression analysis was performed to investigate the possible risk factors for polyautoimmunity in patients with SLE.

Results From all patients included in the registry, 3679 (91.4\%) patients met 4 or more SLE criteria. Of these, 501 (13.6\%) patients had Polyautoimmunity. The characteristics of this group are showed in table 1 . The most frequent polyautoimmunity types associated with SLE were (in descending order over the total cohort of patients with SLE): autoimmune thyroiditis (7.5\%), other connective tissue disorders (4.4\%) and mixed connective tissue disease $(2.7 \%)$. The percentage of patients a family history of SLE was $12.4 \%$.

Multiple autoimmune syndrome was observed in $10.2 \%$ of patients with Polyautoimmunity. The multivariate analysis identified age (odds ratio [95\% confidence interval], 1.01 [1.00-1.02]), sex (3.00 [1.48-6.04]), Raynaud's phenomenon (1.79 [1.342.39]), pulmonary fibrosis (2.88 [1.32-6.30]), Ro-La autoantibodies (1.68 [1.20-2.36]), antiRNP (1.79 [1.32-2.42]) and treatment with methotrexate $(1.54$ [1.08-2.18]) or with antimalarials $(0.57$ $[0.41-0.78])$ as factors associated with polyautoimmunity.

Conclusions SLE patients frequently associate other autoimmune diseases, detecting poliautoimmunity in $14 \%$, MAS in $2 \%$, family history of SLE in $12.4 \%$ and others such as Sjogren's syndrome and secondary SAF in $12.8 \%$ and $12.7 \%$ respectively. More studies are needed to better understand the increase of polyautoimmunity that seems to be observed in SLE.

Funding Source(s): None

\section{DO ALL ANTIPHOSPHOLIPID ANTIBODIES CONFER THE SAME RISK FOR MAJOR ORGAN INVOLVEMENT IN SYSTEMIC LUPUS ERYTHEMATOSUS PATIENTS?}

${ }^{1}$ Leyre Riancho-Zarrabeitia*, ${ }^{2}$ Víctor M Martínez-Taboada, ${ }^{3}$ Iñigo Rua Figueroa, ${ }^{4}$ Fernando Alonso, ${ }^{5}$ Maria Galindo Izquierdo, ${ }^{6} J u a n$ Ovalles-Bonilla, ${ }^{7}$ Alejandro OlivéMarqués, ${ }^{8}$ Antonio Fernandez Nebro, ${ }^{9}$ Jaime Calvo, ${ }^{10}$ Javier Narváez-García, ${ }^{11}$ Eva Tomero Muriel, ${ }^{12}$ Esther Uriarte Isacelaya, ${ }^{13}$ Carlos Galisteo, ${ }^{14}$ Víctor Quevedo Vila, ${ }^{15}$ Enrique Raya, ${ }^{16}$ Javier Narváez-García, ${ }^{17}$ Lorena Expósito, ${ }^{18}$ José A Hernández-Beriaín, ${ }^{19}$ Loreto Horcada, ${ }^{20}$ Jose Maria Pego Reigosa. ${ }^{1}$ Hospital Sierrallana; ${ }^{2}$ Hospital Universitario Marqués de Valdecilla; ${ }^{3}$ Hospital de Gran Canaria dr. Negrin; ${ }^{4}$ Sociedad Española de Reumatología; ${ }^{5}$ Hospital Universitario 12 De Octubre; ${ }^{6}$ Hospital General Universitario Gregorio Marañón; ${ }^{7}$ Hospital German Trias i Pujol; ${ }^{8}$ UGC de Reumatología, Instituto de Investigación Biomédica de Málaga (IBIMA) Hospital Regional Universitario de Málaga, Spain; ${ }^{9}$ Hospital Universitario Araba; ${ }^{10}$ Hospital de Bellvitge; ${ }^{11}$ Hospital De La Princesa; ${ }^{12}$ Hospital De Donostia; ${ }^{13}$ Hospital Parc Taulí. Sabadel; ${ }^{14}$ Hospital Monforte; ${ }^{15}$ San Cecilio Hospital, Granada (Spain); ${ }^{16}$ Bellvitge University Hospital, Barcelona; ${ }^{17}$ Hospital Universitario de Canarias; ${ }^{18}$ Hospital Insular De Gran Canaria; ${ }^{19}$ Complejo Hospitalario de Navarra; ${ }^{20}$ Complexo hospitalario Universitario Vigo

10.1136/lupus-2019-Ism.86 
Abstract 86 Table 1 Association between the different aPL and the number of positive antibodies and APS related features

\begin{tabular}{|c|c|c|c|c|c|c|}
\hline & Arterial thrombosis & Venous thrombosis & Small vessel thrombosis & Fetal death & $\geq 3$ pregnancy losses & Thrombocytopenia \\
\hline LA & $4.45(3.2-6.3) p<0.001$ & $4.9(3.8-6.3) p<0.001$ & $4.7(3.2-7) p<0.001$ & $1.7(1.2-2.5) p<0.001$ & $4.1(2.2-7.3) p<0.001$ & $2.3(1-9-2.8) p<0.001$ \\
\hline aB2GP IgM & $3.5(2.3-5.2) p<0.001$ & $1.8(1.3-2.3) p=0.001$ & $2.7(1.6-4.3) p<0.001$ & $2.2(1.4-3.5) p=0.001$ & $4.6(2.3-9) p<0.001$ & $1.7(1.3-2.2) p<0.001$ \\
\hline aB2GP IgG & $6.5(4.4-9.5) p<0.001$ & $3.2(2.3-4.4) p<0.001$ & $3.7(2.3-5.8) p<0.001$ & $1.8(1.1-2.9) \mathrm{p}=0.024$ & $5.2(2.7-10) p<0.001$ & $1.6(1.2-2.1) p=0.001$ \\
\hline aCL IgM & $2.4(1.8-3.4) p<0.001$ & $2.4(1.9-3.0) p<0.001$ & $4(2.8-5.8) p<0.001$ & $1.7(1.2-2.4) p=0.006$ & $3.1(1.8-5.3) p<0.001$ & $1.8(1.5-2.1) p<0.001$ \\
\hline aCL IgG & $7.3(5.2-10.2) p<0.001$ & $4(3.2-5.0) p<0.001$ & $4(2.8-5.7) p<0.001$ & $1.9(1.4-2.6) p<0.001$ & $3.8(2.2-6.6) p<0.001$ & $1.8(1.5-2.1) p<0.001$ \\
\hline $\mathrm{N}^{\circ}$ of positive antibodies & $2.5(2.2-2.9) p<0.001$ & $2.2(1.9-2.4) p<0.001$ & $2.3(1.9-2.4) p<0.001$ & $1.4(1.2-1.7) p<0.001$ & $2.3(1.8-2.9) p<0.001$ & $1.4(1.3-1.6) p<0.001$ \\
\hline
\end{tabular}

Background Antiphospholipid antibodies (aPL) have been associated with organ damage and certain features in systemic lupus erythematosus (SLE) patients. Our aim is to investigate the association between the different aPL and SLE manifestations as well as to elucidate the influence of the load of antibodies.

Methods Patients from the RELESSER-T registry were included. RELESSER-T is a multicenter, hospital-based registry, with retrospective cross-sectional collection of data from a large representative sample of adult non-selected patients with SLE attending Spanish rheumatology services from the public national health system.

Results Out of a total of 3651 SLE patients, 1368 were positive for aPL (44.9\% of patients were positive for anticardiolipin (aCL) antibodies, $27.3 \%$ for anti b2glycoprotein I (aB2GPI) and 24\% for lupus anticoagulant (LA)). Regarding the load of antibodies, 20.6\%, 12.1\% and 4.8\% were positive for one, two and three antibodies, respectively. The association between the different aPL, the number of positive antibodies and antiphospholipid syndrome related manifestations is showed in table 1 . Overall, all types of aPL were associated with classic APS manifestations, although LA, IgG isotypes, and patients with more than one aPL display a higher risk to develop clinical APS.

Regarding specific lupus manifestations, all aPL types showed a negative association with cutaneous manifestations, and was also significantly associated with the load of autoantibodies $(\mathrm{p}<0.001)$. LA and aCL were associated with an increased risk of cardiac, respiratory and neuropsychiatric manifestations $(p<0.001)$. Furthermore, LA was also associated with an increased risk of renal disease $(p<0.001)$. aCL $\operatorname{IgG}$ was associated with a higher risk of specific lupus manifestations compared with aCL IgM. Interestingly, aB2GP IgG were only associated with an increased risk of seizures $(p<0.001)$. When evaluating the influence of the load of antibodies, we found that the risk of neuropsychiatric manifestations $(\mathrm{p}<0.001)$, as well as the cardiac $(\mathrm{p}=0.003)$, and pulmonary manifestations $(p=0.001)$, significantly increased with a higher number of positive antibodies. Inversely, the risk of cutaneous symptoms decreased while the number of positive antibodies increased (OR 0.89, 95\% CI 0.82-0.96, p=0.003).

Conclusions The present study in a large SLE cohort confirm than there is a hierarchy for aPL and the risk of APS and lupus manifestations. aCL, and especially LA, confer a higher risk for major organ involvement in SLE patients. IgG isotypes and the load of aPL antibodies confer a higher risk for clinical APS and major lupus manifestations.

Funding Source(s): None

\section{MAPPING DISEASE SEVERITY AND PROGRESSION OF RENAL INVOLVEMENT IN PATIENTS WITH SYSTEMIC LUPUS ERYTHEMATOSUS}

${ }^{1} J u s t y n a$ Amelio, ${ }^{2}$ Kerry Gairy, ${ }^{3}$ Anadi Mahajan, ${ }^{3}$ Gavneet Kaur, ${ }^{4}$ Damon Bass, ${ }^{4}$ Rodger Levy*, ${ }^{5}$ David Roth. 'GlaxoSmithKline, Real World Evidence and Epidemiology, Stevenage, Herts, UK; ${ }^{2}$ GlaxoSmithKline, Value Evidence and Outcomes, Brentford, Middlesex, UK; ${ }^{3}$ Bridge Medical, Richmond, London, UK; ${ }^{4}$ GlaxoSmithKline, Immunoinflammation and Future Pipeline, Collegeville, PA, USA; ${ }^{5}$ GlaxoSmithKline, Research and Development, Collegeville, PA, USA

\subsection{6/lupus-2019-Ism.87}

Background Despite recent advances in the treatment of systemic lupus erythematosus (SLE) and lupus nephritis (LN), understanding of their pathogenesis and the interrelation between disease states remains incomplete. A pragmatic review (GSK study LS3178) was conducted to map disease severity and progression of renal involvement in SLE, focusing on: LN development among patients with SLE, within-LN progression, and progression to end-stage renal disease (ESRD).

Methods A keyword based literature search was conducted using PubMed, Google and Google Scholar and supplemented with a bibliography search relevant to the focus area. The following publications were screened and prioritized for inclusion: high quality; published after 2010; addressed a topic of focus or an information gap; data were from the USA or Europe. High-quality pre-2010 and non-USA/Europe publications were permitted.

Results Overall, 248 citations were identified (keyword based search, $n=117$; bibliography search, $n=131$ ). Following full text screening, 144 publications were considered relevant to the review and 26 were selected for inclusion (21 primary studies, 3 narrative reviews and 2 systematic literature reviews). An overview of the results is provided in the Figure. This review identified that $726 \%$ of patients had LN at the time of SLE diagnosis, and $3148 \%$ of patients with SLE developed LN in the disease course, most (8090\%) within 5 years of diagnosis. Class IV nephritis was the most common LN class found at first (3560\%) and repeat (3563\%) biopsy and had the worst prognosis. Histological transformation from one $\mathrm{LN}$ class to another was reported in $4076 \%$ of patients, most commonly in patients with nonproliferative lesions in the first biopsy. Overall, the proportion of patients who subsequently developed ESRD was $36 \%$ (SLE) and $428 \%$ (LN). Limited data existed for time to progression within LN and from SLE/LN to ESRD, and for renal signs present before LN diagnosis. 